\title{
Spectral-acoustic method for outburst danger prediction considering in-situ gas pressure
}

\author{
Alexandr Shadrin ${ }^{1}$, Artyom Kontrimas ${ }^{1}$ \\ ${ }^{1}$ FRC CCC SB RAS. Prosp. Leningradskey, 10, Kemerovo city, 650065, Russian Federation
}

\begin{abstract}
The option of spectral-acoustic method for coal seam outburst danger prediction based on the influence of outburst danger basic factors on the amplitude-frequency characteristic of acoustic vibration median formed when breaking the rock massif by the operating mining equipment is justified. Basic outburst danger factors are: rock pressure, in-situ gas pressure, coal strength.
\end{abstract}

\section{Introduction}

Coal and gas sudden outbursts are dangerous by their consequences. Starting from a certain depth they take place, practically in all coal basins of the world: Russia, Ukraine, China, Kazakhstan, the USA, Australia, Poland etc.

Over many years of researches made by different scientific groups it is stated that coal seam outburst danger depends on a large number of parameters including: rock pressure, insitu gas pressure, filtrating and collecting properties and strength characteristics of a seam, its structure, presence of dislocations, coal humidity, applied technology of the mining etc. $[1-8]$. That is why it is accepted that the prediction should carry multifactorial character $[2$, $3,7,9]$. Based on the analysis the following basic outburst danger factors has been set: zones of the excessive rock pressure (stress state factor) and in-situ gas pressure (gas factor), presence of low strength broken coal bands with sufficient thickness (coal strength factor) where large volumes of sorbed gas are accumulated. As a rule outburst danger zones are confined to the zones of seam structure dislocation of disjunctive and plicative character.

For current outburst danger prediction, controlling methods for basic influencing factors were developed. Initially, instrumental methods (known in Russia under such name) based on the analysis of the basic outburst danger factors on the results of interval drilling of test holes ahead of the workings were developed. Gas factor was estimated according to the initial speed of gas emission from the meter interval of the borehole. Fine yield (drill cuttings) characterized rock pressure and coal strength. The most broken (the weaker) coal band was defined by eye and its strength was measured by a strength measuring device [10]. Resulting from statistical processing of numerous measuring of gas emission initial speed and fine yield the outburst danger criteria were defined by this method for basic coal basins of Russia, Kazakhstan and Ukraine.

The disadvantage of the instrumental method is its significant duration and periodicity (the prediction is fulfilled, as a rule, not more than in 4 meters of development heading advancing). To avoid this disadvantage geophysical prediction methods started to be 
developed. They do not interfere with the mining process. They are based on controlling physical values functionally connected with the basic outburst danger factors, without drilling test holes.

One of such methods is spectral-acoustic one. It was based on the rock pressure influence on amplitude frequency spectrum of the noise generated in a face space by operating mining equipment. The advantage of this method is in continuous character of prediction without sampling coal and gas ahead of the face space. And the "noise" of the operating mechanisms does not interfere with the operation, as in acoustic emission method, but on the contrary, it is used for sounding face space by a broad-band acoustic radiation.

The disadvantage of this method is that it controls only a stress state of a massif sector where the acoustic radiation spreads from the cutting tool of the operating mining equipment till geophone. We'll demonstrate that this method can be supplemented by controlling gas factor and coal strength for increasing the accuracy of the prediction.

\section{Justification of spectral acoustic method of outburst danger prediction considering gas factor}

The justification is in the following: suppose that after the digitalization of an analogue sounding signal and fast Fourier transformation we get a spectrum of $\mathrm{N}$ harmonics. The amplitude of $i$-th harmonic in the distance of $r$ from the source can be written as:

$$
A_{i}(r)=A_{i, 0} F(r) e^{-\omega_{i} r}
$$

where: $A i_{, 0}-$ is an amplitude of $i$-harmonic at the signal source (noise of an operating equipment); $F(r)$ - is a function that considers the diagram of signal source directivity (for example, $F(r)=1$ - for a plane wave; $F(r)=1 / r$ - for spherical wave); $\alpha_{i}$ - the attenuation rate of $i$-harmonic signal; $r$ - the distance between the source and the receiver of the signal.

Assuming that to analyze the state of a face space we analyze the spectrum noise that ranges between $0-1000 \mathrm{HZ}$ and the interval between the neighboring harmonics $\Delta f$ equals, for example, $20 \mathrm{~Hz}$. Then the frequency of $i$-th harmonic will be equal to

$$
f_{i}=20 i, i \in[1]
$$

In first approximation, the attenuation rate $\alpha_{i}$ depends on its frequency $f_{i}$ and current average stresses $\sigma_{c}$ as follows:

$$
\alpha_{i}=\alpha_{0} \beta \frac{f_{i}}{f_{0}} \frac{\sigma_{\mathrm{l}}}{\sigma_{\mathrm{c}}}=\varepsilon f_{i} \frac{\sigma_{\mathrm{I}}}{\sigma_{\mathrm{c}}{ }{ }^{\prime}}
$$

where: $\sigma_{l}$ - is an average limit stress (maximal possible for a controlled section of a seam that precedes the shattering of a massif caused by dynamic phenomenon) on the propagation path of an acoustic signal, $\mathrm{Pa} ; \varepsilon=\frac{\alpha_{0} \beta}{f_{0}}, \mathrm{~m}^{-1} \mathrm{~Hz}^{-1}$.

Then for the selected frequency range the sum of discrete series of amplitudes of an acoustic signal spectral component $A(f, r)$ considering Eqs. (1)-(3) will be equal to:

$$
A(f, r)=\sum_{i=1}^{50} A_{i, 0} F(r) e^{-\varepsilon 20 i \frac{\sigma_{l}}{\sigma_{0}} r}
$$

Assuming that amplitude-frequency characteristic of an acoustic signal at the source is maximal at the central part of the frequency band and its components at the signal transmitter tool can be described by two exponential functions (increasing and decreasing) as follows:

$$
A_{i, 0}=\left\{\begin{array}{c}
A_{0} e^{+\xi 20 i}, i \in[1,14] \\
A_{0} e^{-\eta 20 i}, i \in[15,50]
\end{array}\right\},
$$

where $A_{0}-$ is an amplitude of hypothetic "zero" harmonic with $i=0 \mathrm{~V}$; the parameters $\xi$ and $\eta$-determine the exponents change rate. 
Let us determine parameters $\xi$ and $\eta$ out of the condition that $A_{0}=1,75 \mathrm{~V}$ and amplitudes of the following harmonics satisfy the condition $A_{14,0}=A_{15,0} \approx 1,0 \mathrm{~V}$ with $r=10 \mathrm{~m}$ и $d=\frac{\sigma_{e}}{\sigma_{l}}=1,0$ (the condition of joining of two exponents). Then Eq. (5) is:

$$
A_{i, 0}=\left\{\begin{array}{l}
0.333 \cdot e^{+0.073 i}, i \in[1,14 \\
2.86 \cdot e^{-0.07 i}, i \in[15,50
\end{array}\right\}
$$

Substitute Eq.(6) into Eq.(4). The results of calculating the amplitudes of separate harmonics are introduced in the figure with the following values of input values: $\alpha_{0}=1,3 \mathrm{M}^{-}$ ${ }^{1} ; \beta=0,07 ; f_{0}=500 \mathrm{~Hz} ; F(r)=1 ; r=10 \mathrm{м} ; d=0,2$ и 1,0 .

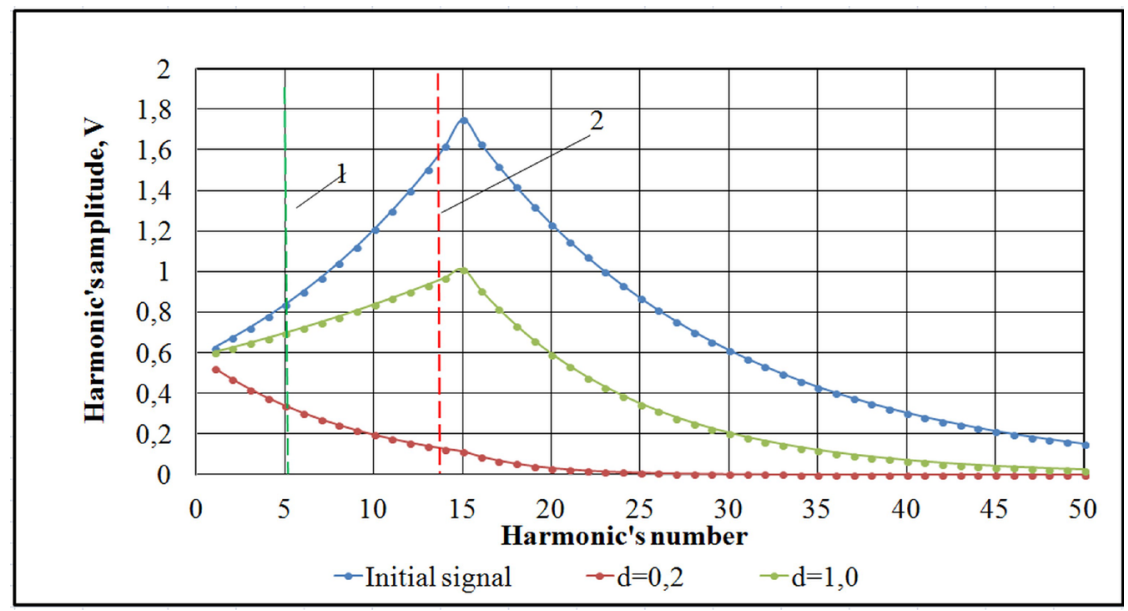

Fig. 1. Amplitude-frequency characteristics of the initial signal that went through the area of a rock massif with the length of $10 \mathrm{~m}$ with the relation of current and limit average stresses $\mathrm{d}=0,2$ and $\mathrm{d}=1,0$ : 1 and 2 are the current values of a median $M_{c}$, equal to $100 \mathrm{~Hz}$ (5-th harmonic) and $260 \mathrm{~Hz}$ (13-th harmonic) for corresponding $d=0.2$ and $d=1.0$.

The Figure demonstrates that together with the stresses growth the amplitudes of highfrequency harmonics increase more significantly than the low-frequency ones. It is also seen that with the growth of the medium current stresses from $0.2 \sigma_{l}$ to $1.0 \sigma_{l}$ the median value increases in 2.6 times starting from the value of $100 \mathrm{HZ}$ and finishing with $260 \mathrm{HZ}$ value.

For introduced parameters of an acoustic signal we will determine the dependence of a current value of discrete series median of spectral component amplitudes $M_{\mathrm{c}}$ on the relation $\frac{\sigma_{E}}{\sigma_{L}}$. Thus, according to the definition median will be understood as the root of equation:

$$
\frac{A_{n}(f, r)}{A_{50}(f, r)}=\frac{\sum_{i=1}^{n} A_{i, 0} F(r) e^{-\varepsilon 20 i \frac{\sigma_{l}}{\sigma_{c}} r}}{\sum_{i=1}^{50} A_{i, 0} F(r) e^{-\varepsilon 20 i \frac{\sigma_{l}}{\sigma_{c}} r}}=0,5
$$

In our case $M_{\mathrm{c}}$ - is a harmonic's frequency value $f_{n}$, where the harmonic amplitude sum from $1^{\text {st }}$ to $n$-th equals the harmonic amplitude sum from $n+1$ to 50 . Then under the assumption that the dependence $F(r)$ for all the harmonics is nearly similar and out of Eq. (7) we have:

$$
\sum_{i=1}^{n} A_{i, 0} e^{-\varepsilon 20 i \frac{\sigma_{l}}{\sigma_{c}} r}=0,5 \sum_{i=1}^{50} A_{i, 0} e^{-\varepsilon 20 i \frac{\sigma_{l}}{\sigma_{c}} r}
$$


As far as the frequencies in a spectrum are given discretely, equation (8) is hard to solve. That is why, in practice, to find median it is reasonable to solve the following inequalities system:

$$
\left.\begin{array}{l}
\sum_{i=1}^{n} A_{i, 0} e^{-\varepsilon 20 i \frac{\sigma_{l}}{\sigma_{c}} r} \leq 0,5 \sum_{i=1}^{50} A_{i, 0} e^{-\varepsilon 20 i \frac{\sigma_{t} r}{\sigma_{c}}} \\
\sum_{i=1}^{n+1} A_{i, 0} e^{-\varepsilon 20 i \frac{\sigma_{l}}{\sigma_{c} r}} \geq 0,5 \sum_{i=1}^{50} A_{i, 0} e^{-\varepsilon 20 i \frac{\sigma_{l} r}{\sigma_{c}} r}
\end{array}\right\}
$$

Let us introduce the concept of relative stresses coefficient $K_{m}$ for the given modification of spectral-acoustic method that equals to the relation of the current value of the median $M_{c}$ to the reference value $M_{0}$ :

$$
K_{m}=M_{\mathrm{c}} / M_{0}
$$

To simplify this, assume that $M_{0}$ corresponds relationship values $d=1.0$. Thus, to define $\mathrm{K}_{\mathrm{m}}$ it is important to know $M_{\mathrm{c}}$ and $M_{0}$. Though if to define the values $\sigma_{c}$ and $\sigma_{l}$ that define them considering in-situ gas pressure and coal strength then $K_{m}$ will be the outburst danger indicator.

Two-stage model for preparing outburst danger situation is introduced. According to the model at the first stage the growth of the fractures in a face space is fulfilled and coal block structure is created. At the second stage several large pieces of coal are squeezed out immediately out of the future cavity mouth and sudden outburst takes place as it is.

For both stages their fulfillment criteria for the option of spectral-acoustic method of prediction where the outburst danger criterion defined through the ratio of high-frequency and low-frequency amplitudes of the noise spectrum parts of the operating mining equipment are defined. Besides in these criteria as far as in the option of the method, studied in the given paper, based on the acoustic-frequency characteristic 'noise' median control, the relation of average limit and current stresses defined considering in-situ gas pressure and coal strength is used. We'll use them in our justification.

Making some mathematical transformations we managed to receive the unified equation for both stages of preparing outburst danger situation that define the relation :

$$
\frac{\sigma_{l}}{\sigma_{c}}=\frac{P_{1}}{0.1\left(\frac{q}{110-q}\right) P_{1}-\Delta D \sqrt{\frac{Q \Omega}{\xi_{i}}}}
$$

where: $P_{1}=1 \mathrm{MPa}$ - normalizing factor, resulting from the expression of the coal strength through non-dimensional indicator $q$ (dimensionless unit), measured by strength measuring device constructed by Skochinsky Institute of mining; $Q$ - air-float rate of the booster fan that delivers the air into the working, $\mathrm{m}^{3} / \mathrm{sec} ; \Omega$ - current value of methane concentration near the working face measured by the methane sensor of gas control apparatus, $\%$; $\xi_{i}$ - coefficient that considers influential level (share of the freshly exposed face area) $i$-th type of the equipment (combined machine, pickhammer, drill bit etc.) on the face, $0<\xi_{i}<1$; In equation (11) $D$ parameter is defined as:

$$
D=m \sqrt{k_{0} \eta P_{a t}} \cdot x_{c r} \exp \left(-\varphi x_{c r}\right) / \sqrt{100 S_{f}}, \mathrm{~Pa} \cdot \sec ^{1 / 2} \mathrm{~m}^{-3 / 2},
$$

where: $m$ - is a constant that characterizes coal porosity; $k_{0}$ is a coefficient of a seam gas permeability, $\mathrm{m}^{-3} ; \eta-$ is a dynamical viscosity of methane, $\mathrm{Pa} \cdot \mathrm{sec} ; P_{a t}-$ gas pressure on the face that equals to atmospheric pressure, $\mathrm{Pa} ; x_{c r}-$ critical distance from the face working where either fracture propagation (for the first stage), or outsqueesing of a coal block (for the second stage of the outburst preparation) is initiated, $\mathrm{m} ; \varphi=1 / x_{s t}-$ constant 
defined by the distance $\left(x_{s t}\right)$ from the face to the section of the massif where the gas pressure is stabilized, $\mathrm{m}^{-1} ; S_{f}$ - a surface area of a face working, $\mathrm{m}^{2} . \Delta$ parameter equals:

$$
\Delta=\max \left\{\frac{\psi_{c r}}{3},(1-\varphi) 10^{-2} \frac{r_{e}}{x_{1}}\right\},
$$

where: $\psi_{c r}\left(x_{c r} / l\right)=k_{1} / k_{1}^{\infty} ; k_{1}$ and $k_{1}^{\infty}$ - are stress intensity coefficients at a point of observation and out of the face working zone influence; $l$ - fracture half-length; $\varphi$ - the coefficient that defines the share of an outsqueezed area along which the fracture edge that separates the section from the rest of the massif, closely contacting; $r_{e}$ and $x_{l}$ - effective radius and the thickness of the outsqueezed coal block, correspondingly.

The analyses shows that the real range values of function arguments $\Delta$ is in the limits from some tenth of 1 to that, close to 2 and they are of commensurable quantities. Then according to Eq. (13) we can approximately take $\Delta=2$.

Parameter $D$ for different stages of preparing the outburst differs by the multiplier $E=x_{c r} \exp \left(-\varphi x_{c r}\right)$, as at the stage of fracture development, $x_{c r}$ corresponds to the distance from the working face to the top of the growing fracture, and at the stage of outsqueezing, value $x_{c r}$ equals to the thickness of an outsqueezed coal block. The fulfilled estimations witness that the numerical values of the given parameter equal, correspondingly: for the stage of fracture development $E \approx 0.1-20.0 \mathrm{~m}$; for the stage of coal block outsqueezing out of the cavity mouth of the developing outburst $E \approx 0.1-0.2 \mathrm{~m}$.

Thus, $D$ parameter which characterizes outburst danger gas factor, for the stage of the fractures growth, changes in a rather large value range then the those for the stage of the coal block outsqueezing, providing that at the little distance from the working face, this parameter is nearly similar for both stages of the outburst preparation.

Substituting Eq. (11) to Eq. (9), we get the inequality system that define the dependence of amplitude-frequency characteristic median of the acoustic "noise" generated by the operating organ of the mining equipment on three basic factors of the outburst danger: rock pressure, in-situ gas pressure and coal strength. In these inequalities $\varepsilon$ and $D$ are unknown variables. To define them experimentally is practically impossible. That is why, it is planned to start the research on the development of the technique for their defining by the way of comparing the results of the simultaneous outburst danger indicator measuring in the controlled working by two methods: spectral-acoustic one which is described in this paper and instrumental one judging by the initial speed of gas emission and fine yield while drilling the controlling borehole.

\section{Conclusion}

The studied option of spectral-acoustic prediction method is based on controlling three basic outburst danger factors: rock pressure, in-situ gas pressure and coal strength.

The idea of the method is in controlling median of amplitude frequency "noise" spectrum formed in the process of breaking coal by mining mechanisms: combined machine, plough-type machine, pickhammer, drilling bit and comparing it with a critical value.

The task for the following researches is in the development of the technique for defining critical value of outburst danger indicator for spectral-acoustic method. 


\section{Acknowledgement}

The research was carried out due to the grant of Russian Science Foundation (project \#1717-01143).

\section{References}

1. Workshop on management and control of high gas emissions and outbursts in underground coal mines (Wollongong, Australia, 2007)

2. Q. X. Yu, Mine gas prevention and control (China University of Mining and Technology, Xuzhou, 1992)

3. C.J. Fan, S. Li, M.K. Luo, W.Z. Du, International Journal of Mining Science and Technology, 1, 49-55 (2017)

4. O. Vardar, C. Zhang, I. Canbulat, B. Hebblewhite, Int. J. Min. Sci. Tech., 28:5, 721-727 (2018)

5. G. Jiabo, X. Jiang, N. Wen, P. Shoujian, Z. Chaolin, L. Xiaohang, Int. J. Min. Sci. Tech., 27:2, 327-332 (2017)

6. D. J. Black, Int. J. Min. Sci. Tech., 27:5, 749-753 (2017)

7. H.W. Zhang, S. Li, Chin. J. Rock Mech. Eng., 24:19, 3577-3581 (2005)

8. A. V. Shadrin, A. A. Kontrimas, IOP Conf. Ser.: Earth Envir. Sci., 84:1, 012040 (2017)

9. A. V. Shadrin, J. Min. Sci., 52:4, 670-682 (2016)

10. A. Shadrin, Y. Diyuk, I. J. Min. Sci. Tech., 29:3, 499-506 (2019) 